\title{
Anomalous Propagation Echo Detection Using Neural Network and Discrete Wavelet Transform
}

\author{
H. Lee, E.K. Kim, S. Kim \\ Department of Electrical and Computer Engineering \\ Pusan National University \\ Republic of Korea
}

\begin{abstract}
Anomalous propagation echo belongs to representative non-precipitation echoes which have very similar characteristics of precipitation echoes. It occurs due to superrefraction or ducting phenomenon of radar beam by temperature or humidity. There are a lot of ongoing researches about detecting and removing the anomalous propagation echo in radar data in order to improve accuracy of data analysis. This paper suggests a detection method with artificial neural network for separating the anomalous propagation echo from radar data, which uses discrete wavelet transform in order to extract characteristics of the anomalous propagation echo. The result derives that artificial neural network with discrete wavelet transform shows good performances.
\end{abstract}

Keywords-anomalous propagation; neural network; discrete wavelet transform

\section{INTRODUCTION}

Weather forecasting is one of important information which affects various fields from personal interests to benefits of companies. It is possible to observe meteorological phenomena using weather radar and satellite due to the technological development. Especially, the weather radar provides essential data of the observed data because the radar has many advantages: fine resolution in space domain; information in near-real time and in remote land areas and over adjacent seas; relatively wide observation range; and so on. Therefore, the analysing process of the radar observation data is essential in weather forecasting.

However, some sorts of unwanted radar echoes are contained in weather radar data such as sea clutter, anomalous propagation, chaff echo, etc. Due to these kinds of unwanted data, which are called non-precipitation echoes, make the forecasting result inaccurate. Therefore, there are a number of researches for quality control (QC) in the forecasting process [1-2]

In this paper, a classification algorithm which uses the artificial neural network is suggested for separating the anomalous propagation echo. Also, in order to extract features of the anomalous propagation echo, discrete wavelet transform is applied. The rest of this paper is as follow. In chapter 2, the features of the anomalous propagation echo explain briefly. And the suggested classification method is described in chapter 3 . After that, the experimental results with the actual anomalous propagation echo are presented in chapter 4 . Finally, the conclusion is showed in chapter 5.

\section{ANOMALOUS PROPAGATION ECHO}

The observation efficiency is different by characteristics of remote sensing device using radio signal. In other words, a beam path of the weather radar is changed by the atmospheric condition. The path is determined by temperature, pressure, distribution of vapour, etc. It is classified as normal refraction, sub-refraction, super-refraction and ducting [3-5] by the path. The types of the refracted radar beam are shown in Figure I .

The weather radar calculates altitude of observation targets consider as the normal refraction of the radar beam. Therefore, unexpected echoes could appear in the observation area of the weather radar by a surface scattering when the super-refraction or the ducting occurs. The surface scattering makes the weather radar to consider trees, mountains, and sea surface as meteorological objects such as rain, snow, etc. The echoes are called as an anomalous propagation echo and the echo generally has strong reflectivity distribution. The anomalous propagation echo gives cause for problems of quantitative precipitation estimation. Hence, there are a number of researches and studies about the anomalous propagation echo in the world [6-7].

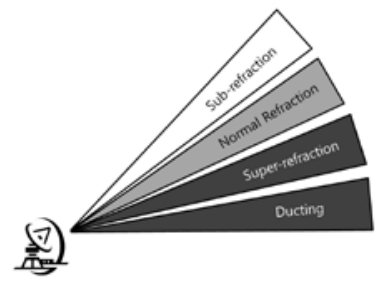

FIGURE I. TYPES OF A REFRACTED RADAR BEAM.

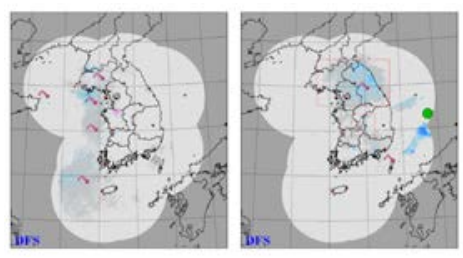

FIGURE II ACTUAL OCCURRENCE CASES OF ANOMALOUS PROPAGATION ECHO.

The actual appearance cases of the anomalous propagation echo are shown in Figure II. The curved arrow marks in Figure II indicate the anomalous propagation echo. Without quality control process by the skilful meteorologist, the observed echoes could be considered as the precipitation echoes. The false weather forecasting by the anomalous 
propagation echo could make economical, material damages in direct or indirect way. Therefore, in order to prevent inaccurate weather forecasting, the anomalous propagation echo should be removed.

\section{CLASSIFICATION METHOD}

In order to obtain accurate weather forecasting result, there are a number of on-going researches in the world. The representative research topics of the anomalous propagation echo are as follow: applying classification algorithm such as fuzzy inference system or Bayesian classifier [8-9]; analysing actual occurrence cases in specific region [10-11]. In this paper, artificial neural network algorithm is chosen as classification method and discrete wavelet transform method is applied for the purpose of extracting characteristics of the anomalous propagation echo.

\section{A. Artificial Neural Network}

The artificial neural network has been considered as an essential and powerful tool for classification due to its advantages. The advantages of artificial neural network are as follow: it is self-adaptive data-driven methods; it could approximate complex functions with arbitrary accuracy; it is flexible in modelling real world complex relationships; it could estimate posterior probabilities which provide the basis for establishing classification rule and performing statistical analysis; and so on [12]. For the purpose of utilizing these advantages, the artificial neural network is selected in this paper.

\section{B. Discrete Wavelet Transform}

The discrete wavelet transform (DWT) provides a transformation of a signal from the time domain to the scalefrequency domain [13]. The DWT is computed on several with different time/scale-frequency resolutions. The scaling function and wavelet function is shown in eqn (1) and eqn (2) where $\mathrm{M}$ indicates the specified number of nonzero coefficient.

$$
\begin{aligned}
\phi(x) & =\sum_{k=0}^{M-1} c_{k} \phi(2 x-k) \\
\psi(x) & =\sum_{k}(-1)^{k} c_{1-k} \phi(2 x-k)
\end{aligned}
$$

The DWT is applied for transforming the reflectivity distribution data into scale-frequency domain. It is possible to decide standard model of the anomalous propagation echo with a lot of actual case studies and statistical approach.

\section{Overall Structures of the Proposed System}

The suggested system of this paper for detecting the anomalous propagation echo is shown in Figure III as a flowchart form. When the raw radar data is generated, coordinate conversion process is applied in order to change the coordinate of radar data from spherical to Cartesian. After that, clustering algorithm is applied in order to make hierarchical structure of the unsorted and converted radar data using the spatial clustering method [14]. Although there are several famous clustering methods such as k-means or fuzzy c-mean, the radar data would not provide the number of cluster. Therefore, the spatial clustering technique which is a kind of agglomerative clustering is applied.

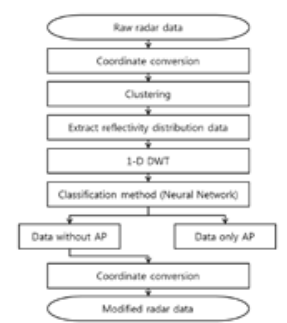

FIGURE III. OVERVIEW OF THE SUGGESTED SYSTEM.

With the clusters which are outputs of the spatial clustering, the reflectivity distribution data is extracted. And the DWT algorithm is applied using the reflectivity distribution data in order to make input of classification method. The classification method is artificial neural network which separates the clusters into the anomalous propagation echo or not. After labelling the echoes, the proposed system generates two kinds of radar data which are the data contain the anomalous propagation echo only and the data without the anomalous propagation echo. Finally, the radar data without the anomalous propagation echo is converted its coordinate for unity of the raw radar data.

\section{EXPERIMENTAL RESULTS}

For the purpose of verification of the suggested system, it was needed to select real occurrence cases of the anomalous propagation echo. Therefore, the actual cases were collected and extracted the reflectivity distribution data both the anomalous propagation echo and the precipitation echo. There were several representative wavelet functions such as Haar, Daubechies, Symlets, Coiflets, and so on. In this paper, Daubechies'4 function was selected because it showed better performance than other functions. The gathered data from the extraction process was separated as training, validation and test data into 7:1.5:1.5. The hidden layer of the artificial neural network was selected as 10 .

Figure. IV indicated the experimental result using the suggested classification method. Except for the small echoes, a large scale echo in centre of the radar image was the anomalous propagation echo. In order to compare the suggested method clearly, two kinds of artificial neural network were implemented: one with discrete wavelet transform; the other without discrete wavelet transform. From the test results, the suggested method showed better result than the without DWT version: $87.1 \%$ accuracy.

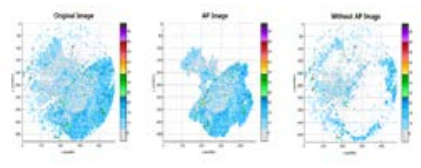

FIGURE IV EXPERIMENTAL RESULT USING ANN AND DWT.

\section{CONCLUSION}

In weather forecasting process, it was greatly important to analyse raw data of weather radar and distinguish precipitation echo and non-precipitation echo. In this paper, in order to detect anomalous propagation echo, the artificial neural network with discrete wavelet transform was suggested as classification method. The proposed classification method 
showed that the proposed classification method can detect the anomalous propagation echo successfully.

Further suggested work is to increase accuracy of separating the anomalous propagation echo. For example, other data could be applied which could be converted from the clusters like maximum reflectivity, radial velocity, altitude, and so on. Also, it is possible to apply suggested classification method to other non-precipitation echoes such as chaff, sea clutter.

\section{ACKNOWLEDGEMENT}

This research was supported by Basic Science Research Program through the National Research Foundation of Korea (NRF) funded by the Ministry of Education(NRF2014R1A1A2056958) and was supported by BK21PLUS, Creative Human Resource Development Program for IT Convergence.

\section{REFERENCE}

[1] Lakshmanan, V., Fritz, T., Hondl, K. \& Stumpf, G., An Automated Technique to Quality Control Radar Reflectivity Data. Journal of Applied Meteorology and Climatology, 11(3), pp. 288-305, 2007.

[2] Steiner, M. \& Smith, J., Use of three-dimensional reflectivity structure for automated detection and removal of non-precipitating echoes in radar data. Journal of Atmospheric and Oceanic Technology, 19, pp. 673-686, 2002.

[3] Fabry, F., Frush, C., Zawadzki, I. \& Kilambi, A., On the extraction of near-surface index of refraction using radar phase measurements from ground targets. Journal of Atmospheric and Oceanic Technology, 14, pp. 978-987, 2002.

[4] Skolnik, M. I., Introduction to Radar Systems 2nd Edition, McGraw-Hill International Editions, pp. 581, 1980.

[5] Jung, S. H., Lee, Y. J. \& Kim, K. E., Investigation of surface duct condition over Korean peninsula, Proceeding of the Autumn Meeting of Korean Meteorological Society, pp. 338-339, 2007.

[6] Cho, H. Y., Lee, G., Kim, K. E. \& Zawadzki, I., Identification and removal of ground echoes and anomalous propagation using the characteristics of radar echoes, Journal of Atmospheric and Oceanic Technology, 23, pp. 1206-1222, 2006.

[7] Hubbert, C. J., Dixon, M., Ellis, S. M. \& Meymaris, G., Weather radar ground clutter. Part I: Identification, Modeling, and Simulation, Journal of Atmospheric and Oceanic Technology, 26, pp. 1165-1180, 2009.

[8] Rico-Ramirez, M. A. \& Cluckie, I. D., Classification of ground clutter and anomalous propagation using dual-polarization weather radar, IEEE Transactions on Geoscience and Remote Sensing, 46(7), pp. 1892-1904, 2008.

[9] Peter, J. R., Seed, A. \& Steinle, P. J., Application of a Bayesian Classifier of Anomalous Propagation to Single-Polarization Radar Reflectivity Data, Journal of Atmospheric and Oceanic Technology, 30(9), pp. 1985-2005, 2013.

[10] Viher, M., Prtenjak, M. T. \& Grisogono, B., A multi -year study of the anomalous propagation conditions along the coast of the Adriatic sea, Journal of Atmospheric and Solar-Terrestrial Physics, 97, pp. 75-84, 2013.

[11] Mesnard, F. \& Sauvageot, H., Climatology of anomalous propagation radar echoes in a coastal area, Journal of Applied Meteorology and Climatology, 49(11), pp. 2285-2300, 2010.

[12] Zhang, G. P., Neural networks for classification: a survey, IEEE Transactions on Systems, Man, and Cybernetics-Part C: Applications and Reviews, 30(4), pp. 451-462, 2000.

[13] Rao, R. M. \& Bopardikar, A. S., Wavelet Transforms-introduction to theory and applications, Addison-Wesley-Longman, 1997.

[14] Kim, Y. H., Kim, S., Han, H., Heo, B. \& You, C., Real-Time Detection and Filtering of Chaff Clutter from Single-Polarization Doppler Radar
Data, Journal of Atmospheric and Oceanic Technology, 30(5), pp. 873865, 2013. 